Z Rheumatol 2021 $80: 771-775$ https://doi.org/10.1007/s00393-021-01000-8 Angenommen: 19. März 2021

Online publiziert: 22. April 2021

๑ Der/die Autor(en) 2021

\section{Redaktion}

M.O. Becker, Zürich

P. Hoff, Berlin

A.J. Hueber, Bamberg

F. Moosig, Neumünster

A. Schramm ${ }^{1}$ D. Windschall ${ }^{2}$ C. Hinze ${ }^{3} \cdot$ D. Föll ${ }^{3} \cdot$ H. Wittkowski $i^{3} \cdot$ H. Winowski ${ }^{2} \cdot$

\title{
S. Oesingmann ${ }^{1}$
}

'Klinik für Kinder- und Jugendmedizin, Allgemeine Pädiatrie, Universitätsklinikum Münster, Münster, Deutschland

${ }^{2}$ Klinik für Kinder- und Jugendrheumatologie, St. Josef-Stift Sendenhorst, Sendenhorst, Deutschland

${ }^{3}$ Klinik für Pädiatrische Rheumatologie und Immunologie, Universitätsklinikum Münster, Münster,

Deutschland

\section{Cushing-Syndrom mit konsekutiver tertiärer} Nebennierenrindeninsuffizienz nach simultaner multipler intraartikulärer Lokaltherapie mit Glukokortikoiden

\section{Bei Mehrfachgabe von lokalen Steroiden behandlungsbedürftige Hormonmangelzustände im Blick behalten}

Die Patientin kam 2,5 Monate später $\mathrm{zu}$ einem vereinbarten stationären Kontrolltermin. Klinisch bestanden bei Aufnahme keine Arthritis und keine Bewegungseinschränkungen, arthrosonographisch wurde im rechten Sprunggelenk und in einigen Fingergelenken noch Restaktivität gesehen. Nach zunächst stabiler Episode hatte die Patientin im Vorfeld ca. 3 Wochen nach der intraartikulären Behandlung einen zunehmend cushingoiden Habitus entwickelt. Intermittierend traten Erbrechen und Abgeschlagenheit auf. Zehn Wochen nach der initialen Gelenkbehandlung und 10 Tage vor der oben genannten Aufnahme war durch die Kinderärztin im Vorfeld bereits eine Labordiagnostik initiiert worden, dabei war Kortisol im Serum $(<0,05 \mu \mathrm{g} / \mathrm{dl}$; Normbereich 4,8-19,5 $\mu \mathrm{g} / \mathrm{dl})$ und Urin $(<0,2 \mu \mathrm{g} / \mathrm{l}$; Normbereich $7-60 \mu \mathrm{g} / \mathrm{l})$ bei morgendlicher Probenentnahme um 9:00 Uhr nicht messbar. Die Patientin stellte sich schließlich mit Fieber vor, und bei Aufnahme zeigte sie einmalig
Erbrechen und einen reduzierten Allgemeinzustand. Das Körpergewicht lag bei $20,9 \mathrm{~kg}$ (91. Perzentile), die Körperlänge bei $106,5 \mathrm{~cm}$ (72. Perzentile) und der Blutdruck bei $96 / 58 \mathrm{~mm} \mathrm{Hg}$ (mittlerer arterieller Druck [MAD] $71 \mathrm{~mm} \mathrm{Hg}$ ). Im Labor zeigten sich erhöhte Entzündungswerte sowie eine Leukozyturie und Proteinurie. Neben einem Harnwegsinfekt wurde ein endokrinologisches Problem als Ursache der Beschwerden vermutet und eine stationäre Abklärung in der Universitäts-Kinderklinik eingeleitet.

\section{Befund}

Bei Aufnahme in der Universitätsklinik fielen ein reduzierter Allgemeinzustand mit hohem Fieber und ein adipöser Ernährungszustand bei cushingoidem Habitus auf (-Abb. 1). Ein spezifischer Infektfokus war klinisch zunächst nicht eruierbar.

Laborchemisch zeigten sich eine

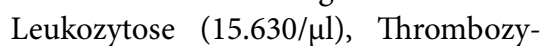
tose $(416.000 / \mu \mathrm{l})$ sowie eine Erhöhung bei sehr großem Appetit. 
Tab. 1 Lokale Glukokortikoidapplikationen an 46 Lokalisationen

\begin{tabular}{|c|c|c|c|}
\hline \multirow{2}{*}{$\begin{array}{l}\text { Links } \\
\text { Gelenk }\end{array}$} & \multirow[b]{2}{*}{$\begin{array}{l}\text { Präparat, } \\
\text { Dosis }\end{array}$} & \multicolumn{2}{|l|}{ Rechts } \\
\hline & & Gelenk & $\begin{array}{l}\text { Präparat, } \\
\text { Dosis }\end{array}$ \\
\hline Schultergelenk & THA $10 \mathrm{mg}$ & Schultergelenk & THA $10 \mathrm{mg}$ \\
\hline Ellenbogengelenk & THA $10 \mathrm{mg}$ & Ellenbogengelenk & THA $10 \mathrm{mg}$ \\
\hline Handgelenk radiokarpal & THA $5 \mathrm{mg}$ & $\begin{array}{l}\text { Handgelenk radiokar- } \\
\text { pal }\end{array}$ & THA $5 \mathrm{mg}$ \\
\hline Handgelenk mediokarpal & THA 0,5 mg & $\begin{array}{l}\text { Handgelenk mediokar- } \\
\text { pal }\end{array}$ & THA 0,5 mg \\
\hline IP-Gelenk & THA 0,5 mg & DS-Gelenk & THA 0,5 mg \\
\hline MCP I & THA $1 \mathrm{mg}$ & $\mathrm{MCPI}$ & THA $1 \mathrm{mg}$ \\
\hline MCP II & THA $1 \mathrm{mg}$ & MCP II & THA $1 \mathrm{mg}$ \\
\hline MCP III & THA $1 \mathrm{mg}$ & MCP III & THA $1 \mathrm{mg}$ \\
\hline MCP IV & THA $1 \mathrm{mg}$ & MCP IV & THA $1 \mathrm{mg}$ \\
\hline MCP V & THA $1 \mathrm{mg}$ & MCP V & THA $1 \mathrm{mg}$ \\
\hline PIP ॥ & THA 0,5 mg & PIP II & THA 0,5 mg \\
\hline PIP III & THA 0,5 mg & PIP III & THA 0,5 mg \\
\hline PIP IV & THA 0,5 mg & PIP IV & THA 0,5 mg \\
\hline PIP V & THA 0,5 mg & PIP V & THA 0,5 mg \\
\hline Hüftgelenk & THA $20 \mathrm{mg}$ & Hüftgelenk & THA $20 \mathrm{mg}$ \\
\hline Kniegelenk & THA $20 \mathrm{mg}$ & Kniegelenk & THA $20 \mathrm{mg}$ \\
\hline Oberes Sprunggelenk & THA $10 \mathrm{mg}$ & Oberes Sprunggelenk & THA $10 \mathrm{mg}$ \\
\hline Talonavikulargelenk & THA $5 \mathrm{mg}$ & Talonavikulargelenk & THA $5 \mathrm{mg}$ \\
\hline ST-Gelenk & THA $5 \mathrm{mg}$ & ST-Gelenk & THA $5 \mathrm{mg}$ \\
\hline CN-Gelenk & THA $5 \mathrm{mg}$ & CN-Gelenk & THA $5 \mathrm{mg}$ \\
\hline Handgelenkstrecksehnen & DEX $1,5 \mathrm{mg}$ & $\begin{array}{l}\text { Handgelenkstreck- } \\
\text { sehnen }\end{array}$ & DEX $1,5 \mathrm{mg}$ \\
\hline Tibialis-posterior-Sehne & DEX $1,5 \mathrm{mg}$ & $\begin{array}{l}\text { Tibialis-posterior-Seh- } \\
\text { ne }\end{array}$ & DEX $1,5 \mathrm{mg}$ \\
\hline Peroneus-brevis-Sehne & DEX $1,5 \mathrm{mg}$ & Peroneus-brevis-Sehne & DEX $1,5 \mathrm{mg}$ \\
\hline $\begin{array}{l}\text { Kumulative Prednisolon-äquivalente } \\
\text { Dosis }^{a}\end{array}$ & $125 \mathrm{mg}$ & - & $125 \mathrm{mg}$ \\
\hline \multicolumn{4}{|c|}{$\begin{array}{l}\text { CN-Gelenk Cuneonavikulargelenk, DEX Dexamethason, DS-Gelenk Daumensattelgelenk, IP-Gelenk In- } \\
\text { terphalangealgelenk, MCP Metakarpophalangealgelenk, PIP proximales Interphalangealgelenk, } \\
\text { PDN Prednisolon, ST-Gelenk Subtalargelenk, THA Triamcinolon-Hexacetonid } \\
\text { aGerundete Prednisolon-äquivalente Dosis in mg gemäß Glukokortikoid-Äquipotenz: Triamcinolon } \\
1 \mathrm{mg}=\text { Prednisolon 1,25 mg (75\% Triamcinolon-Anteil in Triamcinolon-Hexacetonid); Dexamethason } \\
1 \mathrm{mg}=6,25 \mathrm{mg} \text { Prednisolon }\end{array}$} \\
\hline
\end{tabular}

des C-reaktiven Proteins (10,2 mg/dl) als Ausdruck einer deutlichen AkutePhase-Reaktion. Im Urin ließen sich eine Leukozyturie sowie kulturell E. coli nachweisen (Keimzahl 106/ml). Die weitere infektiologische und immunologische Diagnostik blieb unauffällig, ebenso blieben Sonographien von Abdomen und Nieren ohne Hinweis auf eine Harntransportstörung.

Die vorstationären Laborbefunde zeigten Konzentrationen von Kortisol sowohl im Serum als auch Urin unterhalb der Nachweisgrenze trotz des bestehenden Cushing-Syndroms. Am Morgen

\section{Diagnose}

Es wurde ein hochfieberhafter Harnwegsinfekt mit E. coli diagnostiziert. Außerdem bestand ein eindrucksvolles Cushing-Syndrom, vermutlich iatrogen nach intraartikulären Glukokortikoidinjektionen. In Zusammenschau mit den vorstationär nicht nachweisbaren Kortisolspiegeln im Urin und Serum wurde retrospektiv eine konsekutive (tertiäre) Nebennierenrindeninsuffizienz (NNRInsuffizienz) nach exogener Gabe von Steroiden diagnostiziert, die sich jedoch mutmaßlich zum Zeitpunkt der Aufnahme bereits wieder erholt hatte, da nach Aufnahme keine Beeinträchtigung der hypothalamisch-hypophysärkortikotropen Achse mehr nachweisbar war.

\section{Therapie und Verlauf}

Es erfolgte eine antibiotische Therapie mit Cefotaxim, und die MethotrexatGabe wurde pausiert. Da der Zustand der Patientin sich rasch besserte, sie am 3. Tag entfiebert war und die Laborwerte für Kortisol und ACTH eine Erholung der Hypophysen-NebennierenrindenAchse zeigten, konnte auf eine zunächst diskutierte Stressdosisgabe bzw. Substitutionstherapie mit Hydrocortison verzichtet werden. Bei einer ambulanten Nachkontrolle nach 10 Tagen lag das Kortisol basal mit 2,8 ng/ml noch unter der Norm, bei einem normalen ACTH von 11,7 pg/ml. Auf einen ACTHStimulationstest wurde verzichtet. Bei weiterer klinischer Besserung wurde ein abwartendes Vorgehen vereinbart, und ambulante Kontrollen wurden empfohlen. sol basal mit $141 \mathrm{ng} / \mathrm{ml}$ (Norm 11,5-97,5) erhöht und Adrenocorticotropes Hormon (ACTH) lag mit 45,1 pg/ml (Norm 7,3-63,3) im Normbereich. Am Nachmittag (16 Uhr) stieg das ACTH auf $110 \mathrm{pg} / \mathrm{ml}$, um Mitternacht fielen Kortisol $(54 \mathrm{ng} / \mathrm{ml})$ und ACTH $(2,0 \mathrm{pg} / \mathrm{ml})$ im Rahmen der zirkadianen Rhythmik ab. Die übrige endokrinologische Diagnostik (Schilddrüse, Wachstums- und Sexualhormone) blieb unauffällig, der morgendliche Nüchternblutzucker lag mit $94 \mathrm{mg} / \mathrm{dl}$ im Normbereich.

\section{Diskussion}

Die intraartikuläre Glukokortikoidinjektion ist ein gut etabliertes und bewährtes Verfahren in der pädiatrischen Rheumatologie [18]. Trotz vieler Studien an Erwachsenen, die ein signifikantes postinterventionelles Risiko einer NNR-In$20]$, gibt es in der pädiatrischen Literatur nur wenige Daten und ein geringes Bewusstsein für dieses Risiko. suffizienz belegen [5-9, 11, 13-16, 19, 
Die bei Weitem häufigste Ursache einer NNR-Insuffizienz stellt die Pharmakotherapie mit synthetischen Glukokortikoiden dar [9]. Unter dieser Therapie kann es zu einer Suppression der Hypothalamus-Hypophysen-NebennierenAchse mit Atrophie der kortikotropen Zellen der Hypophyse und der NNR kommen (tertiäre NNR-Insuffizienz). Bei Erwachsenen muss unter einer höher dosierten Pharmakotherapie (Prednisolonäquivalent $20-30 \mathrm{mg}$ täglich) bereits nach wenigen Tagen mit einer Suppression des Regelkreises gerechnet werden [17]. Das Risiko einer NNR-Insuffizienz steigt mit der Dosis und Dauer der Therapie. Auch nach Lokaltherapien muss prinzipiell mit der Entwicklung einer NNR-Insuffizienz gerechnet werden. Daten aus Fallserien belegen das Risiko eines Cushing-Syndroms, aber auch der konsekutiven Störung der Hypothalamus-Hypophysen-Nebennieren-Achse nach einzelnen lokalen Steroidinjektionen $[1,2,8,15,20]$. Bei Erwachsenen werden typischerweise $8 \mathrm{~h}$ nach intraartikulären Injektionen Spitzenwerte im Serum erreicht. Die lokal verabreichten Glukokortikoide werden innerhalb von 2 bis 3 Wochen vollständig von der Injektionsstelle resorbiert [19]. Das Absorptionsprofil intraartikulärer Glukokortikoide kann je nach Präparat und Injektionsstelle variieren. In entzündeten Gelenken und in stärker vaskularisierten Bereichen wie den Wachstumsfugen ist die Absorption verstärkt.

Wenn das supprimierende Glukokortikoid reduziert oder abgesetzt wird, können Symptome einer NNR-Insuffizienz oder eines Steroidentzuges auftreten. $\mathrm{Zu}$ den Symptomen einer NNR-Insuffizienz können Müdigkeit, Übelkeit, Erbrechen, Hypotonie sowie eine erhöhte Infektanfälligkeit gehören $[14,16]$. Eine Erholung wird bei den meisten Patienten nach 1 bis 4 Wochen erwartet, kann aber je nach Dosis und Häufigkeit der Injektionen länger dauern $[3,5,7,11,13$, 19]. Bei unserer Patientin gehen wir zum Zeitpunkt der stationären Aufnahme im Rahmen des Harnweginfektes von einer Erholung aus, da sich die gemessenen Werte für die hypothalamisch-hypophysär-kortikotrope Achse hier normalisiert hatten, wir gehen jedoch zu-

Z Rheumatol 2021 ·80:771-775 https://doi.org/10.1007/s00393-021-01000-8

(c) Der/die Autor(en) 2021

A. Schramm · D. Windschall · C. Hinze · D. Föll · H. Wittkowski · H. Winowski · S. Oesingmann

Cushing-Syndrom mit konsekutiver tertiärer

Nebennierenrindeninsuffizienz nach simultaner multipler

intraartikulärer Lokaltherapie mit Glukokortikoiden. Bei

Mehrfachgabe von lokalen Steroiden behandlungsbedürftige Hormonmangelzustände im Blick behalten

\section{Zusammenfassung}

Eine 4-jährige Patientin mit neu diagnostizierter polyartikulärer juveniler idiopathischer Arthritis (JIA) entwickelte nach simultaner multipler Lokaltherapie mit Glukokortikoiden an 46 Stellen zunächst ein CushingSyndrom, gefolgt von einer schleichenden Zustandsverschlechterung und schließlich einem akuten hochfieberhaften Harnwegsinfekt. Dabei wurde eine iatrogene Nebennierenrindeninsuffizienz nach der multiplen intraartikulären Glukokortikoidga- be diagnostiziert. Die Möglichkeit schwerer systemischer Glukokortikoidnebenwirkungen nach ausgedehnten Lokaltherapien sollte in das reguläre Management der JIA-Patienten einbezogen werden.

Schlüsselwörter

Juvenile idiopathische Arthritis · Intraartikuläre Steroidtherapie · Glukokortikoidnebenwirkungen · Nebennierenrindeninsuffizienz Kortisolmangel

\section{Cushing's syndrome with subsequent tertiary adrenocortical insufficiency after simultaneous multiple intra-articular local glucocorticoid treatment. Keep an eye on hormone deficiency conditions requiring treatment after multiple administrations of local steroids}

\section{Abstract}

After simultaneous multiple local treatment with glucocorticoids at 46 sites a 4-yearold female patient with newly diagnosed polyarticular juvenile idiopathic arthritis (JIA) initially developed Cushing's syndrome followed by a gradual worsening of her condition and finally an acute high fever urinary tract infection. latrogenic adrenocortical insufficiency after multiple intraarticular glucocorticoid administration was diagnosed. The possibility of severe systemic glucocorticoid side effects after extensive local treatment should be included in the regular management of JIA patients.

\section{Keywords}

Juvenile idiopathic arthritis - Intra-articular glucocorticoid therapy - Glucocorticoid side effects · Adrenal cortex insufficiency · Cortisol deficiency dem von einer Stressreaktion aus, sodass höhere Werte zu erwarten waren. In einer Studie mit 60 JIA-Patienten wurde ebenfalls eine hohe Prävalenz einer transienten Suppression der Nebenniere gezeigt. Die Speichelkortisolspiegel waren bei 22 Kindern, die eine intraartikuläre Injektion von Triamcinolon-Hexacetonid mit 20-60 mg (meist im Kniegelenk verabreicht) erhielten, über einen Median von 16 Tagen unterdrückt [10]. Keiner der Patienten in dieser Studie entwickelte ein klinisches Cushing-Syndrom, und eine Normalisierung der Kortisolspiegel im Speichel wurde bei der Mehrheit der
Patienten vor Ablauf von 4 Wochen beobachtet.

Die NNR-Insuffizienz kann, wenn sie nicht erkannt wird, zu erhöhter Morbidität und Mortalität führen. Eine angemessene „Stressreaktion“, d.h. die Produktion von ausreichend endogenem Kortisol bei akuter Krankheit oder Trauma, kann erheblich beeinträchtigt sein. Bei unserer Patientin könnte sowohl die Verschlechterung des Allgemeinzustandes als auch die Krankheitslast des Harnwegsinfektes durch diese Problematik erklärt werden. In der behandelnden kinderrheumatologischen Einrichtung war trotz großem Kollektiv intraartikulär be- 

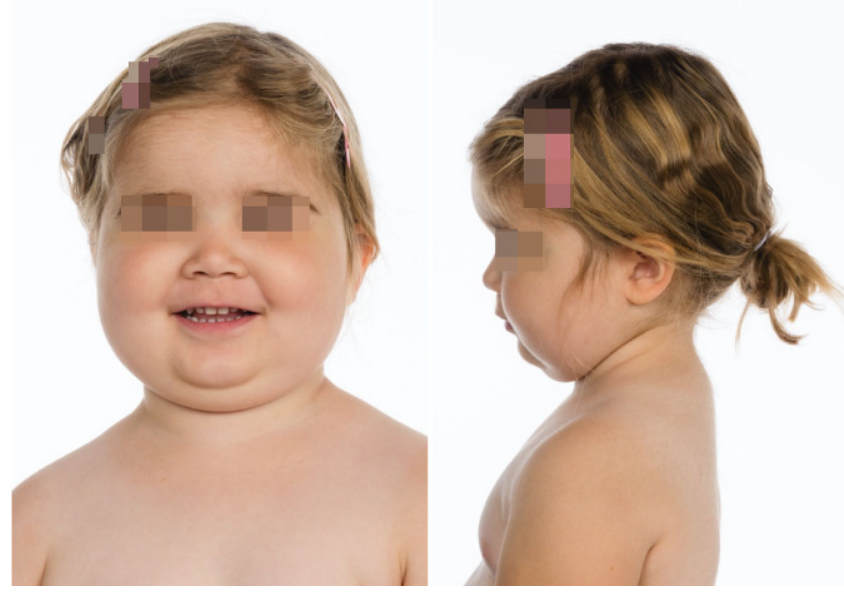

Abb. $1<$ Befund des Cushing-Syndroms bei der Patientin. Es zeigte sich die typische Konstellation eines Cushing-Syndroms mit Mondgesicht, Hirsutismus an Rücken, Stirn und über der Oberlippe undadipöserHypertrophie der Mammae beidseits ohne Brustdrüsengewebe

handelter Patienten ein derartiger Fall noch nicht aufgefallen. Während die Entwicklung eines Cushing-Syndroms nach lokaler Glukokortikoidtherapie gut belegt ist, finden sich in der Literatur kaum Berichte über gravierende Probleme aufgrund einer temporären Unterdrückung der Hypothalamus-Hypophysen-Nebennieren-Achse bei Kindern nach lokaler Glukokortikoidgabe [12, 20]. Das Risiko eines Steroidentzuges ist allerdings allgemein bekannt. Aus diesem Grund wird die orale Glukokortikoidtherapie üblicherweise langsam ausgeschlichen. Bei intraartikulärer Gabe von Steroidpräparaten ist ein kontrolliertes Ausschleichen nicht möglich, was potenziell zu erheblichen Spitzen mit hohen Kortisolspiegeln in den ersten Wochen nach Injektion und anschließend niedrigen Werten in der Folgezeit führen kann. Diese Problematik ist bei multipler simultaner Anwendung an zahlreichen Körperstellen von noch größerer Relevanz. In einem kürzlich veröffentlichten Umfrageergebnis unter weltweit 358 Kinderrheumatologen wurde die Nebennierenrindeninsuffizienz als mögliche Komplikation nicht dokumentiert. In dieser Umfrage zeigten sich eine große Varianz und geringe Standardisierung der Prozeduren, sowohl was das technische Verfahren, den Einsatz von Ultraschall, die Anästhesie, die Wahl der Medikamente als auch ihre Dosierungen angeht [4]. Es gibt keine evidenzbasierten Empfehlungen zu einer Maximaldosis oder -anzahl bei Mehrfachpunktionen, aber überwiegend werden einzelne oder we- nige Gelenke injiziert, der Median lag in der Umfrage bei 4 Gelenken pro Sitzung, aber es gab auch Ausreißer mit einem Maximum von 45 simultan injizierten Gelenken. Der Vorteil der lokalen Glukokortikoidapplikation in Kombination mit einer entlastenden Gelenkpunktion liegt in höheren lokalen Wirkstoffspiegeln an den betroffenen Körperstellen und einem raschen Wirkungseintritt. Ein weiterer Vorteil ist eine längere lokale Wirksamkeit aufgrund der verzögerten Glukokortikoidresorption. Alternativ wäre eine perorale oder intravenöse systemische Brückentherapie mit Glukokortikoiden möglich gewesen. Bei einer peroralen Glukokortikoidtherapie wäre eine Narkoseintervention verzichtbar gewesen. Der Nachteil gegenüber der intraartikulären Therapie ist eine geringere Effektivität sowie eine höhere Tablettenlast mit gastrointestinalen Nebenwirkungen. Auch bei systemischer Gabe von Glukokortikoiden kann es zu einem iatrogenen Cushing-Syndrom kommen, der Hauptvorteil liegt aber in der guten Steuerbarkeit gerade in der Ausschleichphase.

\section{Fazit}

Angesichts der weit verbreiteten Anwendung intraartikulärer Glukokortikoidinjektionen und ihrer klinischen Wirksamkeit müssen sich Ärzte, die diese Injektionen verabreichen, über die potenziellen Risiken des iatrogenen Cushing-Syndroms sowie einer konsekutiven Störung der Hypothalamus-Hypophysen-Nebennieren-Achse im Klaren sein. Patienten und Familien können bei hohen kumulativen Dosen über die Risiken und die Symptome einer Nebennierenrindeninsuffizienz aufgeklärt werden. Die Betreuung der Patienten nach Intervention sollte risikoadaptierten Standards folgen. $\mathrm{Pa}$ tienten können im Bedarfsfall mit oralen Glukokortikoiden supplementiert werden, besonders wenn sie ein Trauma erleiden, sich einem chirurgischen Eingriff unterziehen müssen oder akut erkranken. Weitere Studien im pädiatrischen Patientenkollektiv sollten zu dieser Thematik erfolgen.

\section{Korrespondenzadresse}

\section{PD Dr. med. H. Wittkowski}

Klinik für Pädiatrische Rheumatologie und Immunologie, Universitätsklinikum Münster Domagkstr. 3, 48149 Münster, Deutschland helmut.wittkowski@ukmuenster.de

Funding. Open Access funding enabled and organized by Projekt DEAL.

\section{Einhaltung ethischer Richtlinien}

Interessenkonflikt. A. Schramm, D. Windschall, C. Hinze, D. Föll, H. Wittkowski, H. Winowski und S. Oesingmann geben an, dass kein Interessenkonflikt besteht.

Für diesen Beitrag wurden von den Autoren keine Studien an Menschen oder Tieren durchgeführt. Für die aufgeführten Studien gelten die jeweils dort angegebenen ethischen Richtlinien. Für Bildmaterial oder anderweitige Angaben innerhalb des Manuskripts, über die Patienten zu identifizieren sind, liegt von innen und/oder ihren gesetzlichen Vertretern eine schriftliche Einwilligung vor.

Open Access. Dieser Artikel wird unter der Creative Commons Namensnennung 4.0 International Lizenz veröffentlicht, welche die Nutzung, Vervielfältigung, Bearbeitung, Verbreitung und Wiedergabe in jeglichem Medium und Format erlaubt, sofern Sie den/die ursprünglichen Autor(en) und die Quelle ordnungsgemäß nennen, einen Link zur Creative Commons Lizenz beifügen und angeben, ob Änderungen vorgenommen wurden.

Die in diesem Artikel enthaltenen Bilder und sonstiges Drittmaterial unterliegen ebenfalls der genannten Creative Commons Lizenz, sofern sich aus der Abbildungslegende nichts anderes ergibt. Sofern das betreffende Material nicht unter der genannten Creative Commons Lizenz steht und die betreffende Handlung nicht nach gesetzlichen Vorschriften erlaubt ist, ist für die oben aufgeführten Weiterverwendungen des Materials die Einwilligung des jeweiligen Rechteinhabers einzuholen. 
Weitere Details zur Lizenz entnehmen Sie bitte der Lizenzinformation auf http://creativecommons.org/ licenses/by/4.0/deed.de.

\section{Literatur}

1. Cheng OT, Souzdalnitski D, Vrooman B et al (2012) Evidence-based knee injections for the management of arthritis. Pain Med 13:740-753

2. Cole BJ, Schumacher HR Jr. (2005) Injectable corticosteroids in modern practice. J Am Acad Orthop Surg 13:37-46

3. Derendorf H, Mollmann H, Gruner A et al (1986) Pharmacokinetics and pharmacodynamics of glucocorticoid suspensions after intra-articular administration. Clin Pharmacol Ther 39:313-317

4. Dhanrajani A, Khubchandani RP (2020) Joint injection practice variations in pediatric rheumatology - a global survey and call for action. Pediatr Rheumatol Online J 18:81

5. Duclos M, Guinot M, Colsy M et al (2007) High risk of adrenal insufficiency after a single articular steroid injection in athletes. Med Sci Sports Exerc 39:1036-1043

6. Gondwe JS, Davidson JE, Deeley S et al (2005) Secondary Cushing's syndrome in children with juvenile idiopathic arthritis following intraarticular triamcinolone acetonide administration Rheumatology 44:1457-1458

7. Habib GS (2009) Systemic effects of intra-articular corticosteroids. Clin Rheumatol 28:749-756

8. Hameed R, Zacharin MR (2006) Cushing syndrome, adrenal suppression and local corticosteroid use. JPaediatr Child Health 42:392-394

9. Henzen C, Suter A, Lerch E et al (2000) Suppression and recovery of adrenal response after shortterm, high-dose glucocorticoid treatment. Lancet 355:542-545

10. HuppertzHI,PfullerH (1997)Transient suppression of endogenous cortisol production after intraarticular steroid therapy for chronic arthritis in children. JRheumatol 24:1833-1837

11. Lazarevic MB, Skosey JL, Djordjevic-Denic G et al (1995) Reduction of cortisol levels after single intra-articular and intramuscular steroid injection. Am JMed 99:370-373

12. Levin C, Maibach HI (2002) Topical corticosteroidinduced adrenocortical insufficiency: clinical implications. Am JClin Dermatol 3:141-147

13. Mader R, Lavi I, Luboshitzky R (2005) Evaluation of the pituitary-adrenal axisfunction following single intraarticular injection of methylprednisolone. Arthritis Rheum 52:924-928

14. O'sullivan MM, Rumfeld WR, Jones MK et al (1985) Cushing's syndrome with suppression of the hypothalamic-pituitary-adrenal axis after intra-articular steroid injections. Ann Rheum Dis 44:561-563

15. PekarekB, OsherL, Buck Setal (2011) Intra-articula corticosteroid injections: a critical literature review with up-to-date findings. Foot 21:66-70

16. Reid DM, Eastmond C, Rennie JA (1986) Hypothalamic-pituitary-adrenal axis suppression after repeated intra-articular steroid injections. Ann Rheum Dis 45:87

17. Schlaghecke R, Kornely E, Santen RT et al (1992) The effect of long-term glucocorticoid therapy on pituitary-adrenal responses to exogenous corticotropin-releasing hormone. N Engl J Med 326:226-230

18. Scott C, Meiorin S, Filocamo G et al (2010) A reappraisal of intra-articular corticosteroid therapy in juvenile idiopathic arthritis. Clin Exp Rheumatol 28:774-781

19. Weitoft T, Ronnblom L (2006) Glucocorticoid resorption and influence on the hypothalamic-pituitary-adrenal axis after intra-articular treatment of the knee in resting and mobile patients. Ann Rheum Dis 65:955-957

20. Wojcik M, Ruszala A, Janus D et al (2019) Secondary adrenal insufficiency due to intraarticular glucocorticoid injections. Indian Pediat 56:242-243

\section{MEditorial
Manager}

\section{Hilfestellungen für den Editorial Manager}

Das Einreichungs- und Begutachtungssystem Ihrer Zeitschrift

Sowohl für die ganz alltäglichen Fragen in der Handhabung des Editorial Managers als auch für spezielle Problematiken finden Sie auf www.springermedizin.de/editorialmanager eine Vielzahl an Handreichungen, die Ihnen die Arbeit als Gutachter*in, Autor*in oder Herausgeber*in erleichtern.

Über Videos, einseitige Schritt-für-SchrittAnleitungen oder ein umfangreiches Manual werden Sie durch die einzelnen Punkte geführt, wie:

- Wie reiche ich ein Manuskript ein?

- Wie finde ich passende Gutachter*innen?

- Wie lade ich Gutachter*innen ein?

- Wie nehme ich ein Gutachten an bzw. lehne es ab?

- Wo erkenne ich, in welchem Status ein Mansukript ist?

- Wie ändere ich meine persönlichen Informationen?

- Wo kann ich meinen Urlaub eintragen?

Zugang auch über QR-Code:

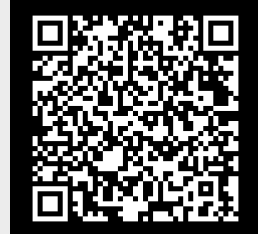

